\title{
Reacciones del consumidor mexiquense hacia las faltantes en anaquel ¿Qué factores intensifican las reacciones negativas hacia el detallista?
}

Victoria Eugenia

\section{Erosa Martín}

Unidad Académica Multidisciplinaria de Comercio y Administración Victoria, Universidad Autónoma de Tamaulipas erosav@usa.net

\section{María del Pilar Ester}

Arroyo López

Departamento de Ciencias Administrativas y Mercadotecnia, Escuela de Negocios, Instituto Tecnológico y de Estudios Superiores de Monterrey, campus Toluca

pilar.arroyo@itesm.mx

\section{Resumen}

Este estudio analiza las reacciones de los consumidores ante faltantes de anaquel en una empresa local del sector del comercio al menudeo con el propósito de apoyar en el diseño de estrategias para incrementar la satisfacción y retención de su clientela. Se tomaron como referencias teóricas la satisfacción y el comportamiento del consumidor; para ello, se realizó una encuesta en tiendas detallistas con distintos perfiles donde se recopilaron datos sobre el perfil demográfico y de compra de los consumidores, así como sus reacciones ante faltantes en la tienda. Cabe aclarar que los datos se analizaron a través de varias técnicas estadísticas. Se concluye que el género, lugar de residencia del cliente, valor de la compra y su lealtad a la tienda afectan significativamente su intención de cambiarla cuando ésta registra faltantes de los productos de su lista de compra. Los resultados obtenidos identifican no sólo segmentos de consumidores, sino también tipos de tiendas más sensibles a sufrir un deterioro en su base de clientes ante la ocurrencia de faltantes.

Palabras clave: faltantes en anaquel, tiendas detallistas, conducta del consumidor. 


\title{
Reactions of State of Mexico consumers when facing out-of-stocks: What fac- tors intensify the negative reactions toward the retailer?
}

\begin{abstract}
This study analyzes the reactions of customers to out-of-stocks in retailing stores, with the objective of supporting the design of strategies to increase customer retention and satisfaction. The theoretical framework was that of customer satisfaction and customer behavior, and a survey was applied at retail stores of distinctive profile to gather data of consumers' demographic and buying profiles along with behavioral reactions under an out-of-stock situation. The collected data was analyzed with the use of multiple statistical techniques. The analysis allows concluding that customers' gender, residence location, ticket value and store loyalty had a significant effect on their intention to switch to another store when some of the products in their shopping list are out of stock. The analysis of results not only identified customer segments but also types of stores more susceptible to being affected in their client base by the occurrence of out-of-stocks.
\end{abstract}

Keywords: out-of-stock, retailers, consumer behavior.

\section{Introducción, referente teórico e hipótesis}

En el sector de venta al menudeo, la ocurrencia de faltantes en anaquel (oos, out of stock, por sus siglas en inglés) es un problema persistente a pesar de los esfuerzos de proveedores y tiendas minoristas por implementar estrategias para sincronizar el abasto, empatar la producción con la demanda y asegurar la entrega de productos en tiempo y costo. Entre estas estrategias figuran Quick Response (QR), ECR (Efficient Consumer Response) y CPFR (Collaborative Planning, Forecasting and Replenishment) y otras iniciativas específicas a la díada detallista-proveedor como SAM (Shelf Availability Monitor) o Item Velocity Monitor. Sin embargo, los avances tecnológicos utilizados para automatizar el abasto, mejorar la administración del inventario, perfeccionar los estimados de la demanda usando datos del punto de venta (POS) han visto contrarrestado su efecto ante el aumento en la cantidad de productos, el incremento en promociones y en las mezclas de productos (marketing mix) que se ofrecen para atender a consumidores con distintos perfiles (Angerer, 2004; Gruen y Corsten, 2008).

Los estudios relacionados con oos realizados en varios países y, en particular, los reportes de Gruen y Corsten $(2002,2008)$ estiman la tasa de faltantes en el nivel 
mundial en un $8.3 \%$, con una mayor incidencia de oos en Europa (8.6\%) que en Estados Unidos (7.9\%). Estas mismas fuentes indican que la cifra varía considerablemente según la cadena detallista y la tienda de autoservicio, en la mayoría de los casos la tasa está entre 5-10\%. La presencia de oos se atribuye principalmente a: 1) las prácticas deficientes en la cadena detallista en especial al proceso de colocación de órdenes ya sea porque éstas son insuficientes o tardías (13-19\% de los casos); 2) los pronósticos inadecuados para la demanda (35-50\% de los casos); 3 ) la mala planeación del abasto ante una promoción vigente o cuando un artículo es descontinuado (25\%); y 4) al resurtido ineficiente de los anaqueles con los productos ya disponibles en el almacén de la tienda (25\%) (Gruen y Corsten, 2008; Van Woensel et al., 2007).

En materia de productos agotados, en Estados Unidos se ha identificado que la tasa de faltantes es mayor entre los productos en promoción (17.1\% versus $7.9 \%$ para productos regulares), donde las categorías con menor número de SKUs (Stock Keeping Units = unidades de guardado en inventario) y las marcas de bajo movimiento y/o escasa preferencia, por parte del consumidor, son las que registran un menor porcentaje de oos. Por otra parte, los productos no-perecederos (abarrotes) al ser ordenados vía electrónica registran menos faltantes que los perecederos que tienden a ordenarse manualmente (van Woensel et al., 2007). Asimismo, se ha reportado un incremento en el porcentaje de oos durante domingos y lunes (10.9\%), mejora el abasto durante viernes y sábado (7.3 y 8.7\%) (Gruen y Corsten, 2008) debido a que el resurtido se realiza en los días y horario en que se prevé mayor demanda (Van Woensel et al., 2007).

Los OOS tienen un efecto relevante sobre la planeación de surtidos, la asignación de espacio en anaquel y la logística, lo que da lugar a una línea de investigación en administración del abasto, la cual está fuertemente vinculada con la disciplina de administración de la cadena de abastecimiento (Chopra y Meindl, 2004). Otra línea de investigación vinculada con oos se ha enfocado en la evaluación de su impacto sobre la satisfacción y la lealtad del cliente. Autores como Verbeke et al. (1998), Kristensen et al. (2001), Angerer (2004), Corsten y Gruen (2004) y Gruen y Corsten (2008) consideran que la disponibilidad de una mezcla de surtidos es un factor que incide directamente en la satisfacción y la lealtad del cliente. Estudios como el de Broniarczyk et al. (1998), Krum (1994) y Dreze et al. (1994) muestran que existe un umbral de tolerancia a las faltantes de productos siempre que éstas ocurran en marcas que no sean las de mayor demanda. Mientras no se exceda este umbral, el desabasto de productos secundarios no tiene un efecto sustancial en la 
actitud de los clientes hacia las tiendas detallistas. Sin embargo, estos estudios también reconocen que el bajo impacto del oos en ventas inmediatas no garantiza un impacto de largo plazo sobre la preferencia hacia una tienda como resultado de la disminución en las percepciones del consumidor con respecto a la calidad de la oferta de surtidos.

Estudios como los de Verbeke et al. (1998) y Gruen y Corsten (2008) señalan que el porcentaje de consumidores totalmente satisfechos con el servicio de una tienda de venta al menudeo depende de:

a) La disponibilidad de productos. A mayor porcentaje de oos menor porcentaje de clientes satisfechos.

b) El número de productos que adquiere el cliente. A menor cantidad de productos requeridos menor el porcentaje de clientes satisfechos cuando un producto deseado no está disponible. Por ejemplo, para una disponibilidad de productos del $90 \%$, el porcentaje de clientes totalmente satisfechos es de $81 \%$ cuando el cliente demanda 20 productos, mientras que asciende a $96 \%$ cuando el cliente compra 40 productos. De donde se infiere que las tiendas enfocadas a compras de conveniencia resienten más el efecto de las oos.

c) La lealtad de los clientes a la marca. Entre mayor la lealtad a una marca particular mayor la insatisfacción del consumidor con la tienda detallista y mayor su propensión a cambiar de tienda para obtener la marca deseada (Retail Insight, 2009).

Este estudio enfoca el impacto de oos sobre el desempeño de tiendas detallistas del tipo supermercado y conveniencia desde la perspectiva de las reacciones del consumidor cuando se encuentra con faltantes en anaquel, independientemente de las causas que la originan y de factores adicionales que contribuyen a la satisfacción del cliente como es la calidad del servicio, la ubicación o el diseño de la tienda de autoservicio. El interés por esta clase de cadenas detallistas se justifica en la importancia que este sector tiene para la economía del país (14\% del PIB nacional se asocia al sector comercio y en la necesidad de proponer alternativas para la recuperación de las ventas en los establecimientos minoristas, las cuales pasaron de crecer un $10.6 \%$ en 2004 con respecto a 2003 a decrecer un $4.6 \%$ en 2009 con relación a 2008 (datos del INEGI). El conocimiento de las reacciones del consumidor ante faltantes de anaquel es relevante para definir la estrategia de mercado y asegurarse de proporcionar aquella mezcla de productos que mejor satisface al consumidor. Las preguntas de investigación planteadas en este estudio son las siguientes: ¿cuál 
es el perfil del consumidor (demográfico, económico y de compra) más proclive a la sustitución? y ¿qué características de la tienda detallista inducen a una mayor sustitución en caso de ocurrencia de faltantes?

En cuanto a la satisfacción del consumidor, se señala que determinados atributos del producto/servicio clasificados como básicos son requisitos previos que el cliente da por descontados y que de no estar presentes generan insatisfacción (Kano et al., 1984). En este estudio, la disponibilidad del producto se considera uno de estos elementos básicos generadores de insatisfacción, la cual a su vez influye sobre la conducta del cliente. Para evaluar las consecuencias que las faltantes tienen sobre fabricantes y tiendas detallistas se han utilizado los cambios en el comportamiento del cliente hacia la presencia de oos. Las cinco reacciones de corto plazo más frecuentemente registradas son: 1) comprar el artículo en otra tienda que ofrezca un surtido similar; 2) retrasar la compra del artículo para otra visita a la misma tienda; 3) sustituir el artículo por otro de la misma marca, pero en otra presentación o con distintas características; 4) sustituir el artículo por otro de una marca diferente pero equivalente en sus características; o 5) cancelar la compra del artículo (Erosa y Arroyo, 2003; Gruen y Corsten, 2002; Verbeke et al. 1998).

Las reacciones varían dependiendo de la categoría del producto, se observó que entre el $21-43 \%$ de los clientes optan por comprar en otra tienda cuando no localizan los productos deseados, mientras que entre el $7-25 \%$ prefiere ya no realizar la compra del producto. A partir de esta información se estima que las tiendas de venta al menudeo pierden alrededor del $40 \%$ de las compras proyectadas debido a la ocurrencia de faltantes, en tanto que los fabricantes pierden un $35 \%$; esto equivale en promedio a un $3.3 \%$ y $2.7 \%$, respectivamente, de ventas perdidas (recuérdese que la tasa de referencia de oos es $8.3 \%$ ). Pero el efecto de las oos no sólo incluye el costo asociado a la pérdida en ventas, sino también el costo asociado a la pérdida en clientes, costo que en el largo plazo tiene consecuencias aún más graves para el detallista y el fabricante (Erosa y Arroyo, 2003).

Estudios realizados en varios países concluyen que la ocurrencia crónica de faltantes tiene como resultado un deterioro en la lealtad del cliente hacia marcas y tiendas detallistas (Gruen y Corsten, 2002; Erosa y Arroyo, 2003; Corsten y Gruen, 2004). Aquellas tiendas y proveedores con menor nivel de oos pierden menos clientes y logran captar los clientes de las tiendas y marcas con mayor incidencia de faltantes. La sustitución de productos ya sea por otros de la misma marca o de marcas competidoras es la reacción que menos afecta a la tienda detallista, 
por lo que es relevante identificar las características de los consumidores menos propensos a esta conducta porque esto permite a las cadenas detallistas concentrar sus esfuerzos de reducción de oos en aquellas tiendas que sirven a consumidores menos propensos a la sustitución y más atraídos a favorecer otra tienda cuando se enfrentan a faltantes de categorías críticas.

Las reacciones del consumidor ante oos se han relacionado con sus características personales (demográficas y económicas), su patrón de compra (e.g. frecuencia de compra y horario de compra), las características del producto que no localizó en anaquel (situación de consumo y categoría del producto), su grado de lealtad a la marca, lealtad a la tienda y el valor de su ticket de compra. De acuerdo con los estudios de Verbeke et al. (1998), de van Woensel et al. (2007) los factores antes citados inciden de manera significativa sobre las reacciones del cliente; por ejemplo, cuando el consumidor es cliente ocasional de la tienda (baja lealtad) o el monto de sus compras es bajo, es más probable que decida comprar el faltante en otra tienda, en tanto que si el consumidor compra con alta frecuencia en la tienda (cliente leal) es más probable que aplace la compra del producto faltante para otra visita o sustituya marcas de productos dentro de la misma tienda. La discusión anterior lleva a formular las siguientes hipótesis:

H1: Ante la presencia de faltantes, los consumidores exhiben diferentes conductas (sustitución, posponer compra o comprar en otra tienda) dependiendo de su perfil demográfico (edad, género, vivir solo o acompañado y lugar de residencia)

$\mathrm{H} 2$ : Ante la presencia de faltantes, los consumidores con menor valor de su ticket de compra son más propensos a realizar la compra de faltantes en otra tienda detallista.

H3: Ante la presencia de faltantes, los consumidores con mayor lealtad a la tienda son menos propensos a comprar el faltante en otra tienda detallista.

Por otra parte, cuando el costo de sustituir el producto es alto - como ocurre con productos de uso personal tales como cosméticos o de higiene personal—o se trata de productos cuyo consumo no se puede diferir fácilmente - como en el caso de productos perecederos - es más frecuente que el cliente decida adquirir el producto en otro establecimiento, sobre todo en aquellos casos en que el costo de cambiar de tienda se percibe bajo; por ejemplo, para productos en la categoría de "belleza y salud" entre el 40-60\% de los clientes opta por adquirirlos en otros establecimiento 
cuando no están disponibles. Ante la tendencia de las cadenas detallistas a ubicar sus tiendas en zonas de fácil acceso o de alta dinámica comercial en las cuales operan tiendas de cadenas competidoras, el costo de cambiar a otro supermercado es cada vez más bajo, lo cual incrementa el riesgo de pérdida de consumidores ante oos. Esto lleva a formular la siguiente hipótesis:

H4: Ante la presencia de faltantes y si hay tiendas cercanas con un surtido similar, los consumidores tienden más a cambiar de tienda detallista.

De acuerdo con Verbeke et al. (1998) y Gruen y Corsten (2008) las compras de bajo monto (bajo valor del ticket de compra o compra de unos pocos productos) se relacionan con una mayor frecuencia de compras en pequeñas tiendas detallistas y con la realización de compras en un mayor número de tiendas. Estas observaciones sugieren que para las tiendas de conveniencia el impacto de oos es más fuerte porque los consumidores tienden a la variedad al comprar en múltiples establecimientos y a adquirir sólo unos cuantos productos para consumo inmediato. De aquí resulta la última hipótesis planteada en este trabajo:

H5: Los clientes de establecimientos de venta al menudeo del tipo tienda de conveniencia son más propensos a comprar en otra tienda cuando encuentran productos faltantes, que los consumidores que realizan compras en supermercados que ofertan un surtido completo de productos.

\section{Metodología}

Para responder a la pregunta de investigación y validar las hipótesis planteadas previamente, se aplicó un cuestionario entre clientes de una cadena de venta al menudeo que opera en el nivel regional en la zona centro del Estado de México y que cuenta con dos tipos de tiendas: 1) autoservicios, que ofrecen un surtido completo de productos similar al de cadenas de supermercados con cobertura nacional; y 2) tiendas de conveniencia con un horario comercial hasta las 21 horas y un surtido de productos que incluye productos frescos. Este estudio se enfoca exclusivamente a estos tipos de tiendas detallistas, se excluyen otras categorías como las tiendas departamentales.

Para verificar las hipótesis H4 y H5 se consideraron dos factores: la existencia de competencia para la tienda dentro de su área de influencia y el tipo de surtido que ofrece. Al combinar estos factores se definieron los siguientes cuatro perfiles o 
categorías de tiendas: 1) tiendas con surtido completo localizadas dentro de zonas comerciales donde se ubican otras tiendas detallistas con una mezcla de surtidos similar y que serán identificadas como tiendas A; 2) tiendas con surtido completo relativamente aisladas (otro supermercado similar se ubica a más de $2 \mathrm{kms}$.) identificadas como tiendas $\mathrm{B}$; 3) tiendas de conveniencia con competencia en el área denotadas como tiendas $\mathrm{C}$; y 4) tiendas de conveniencia sin competidores cercanos (la tienda de conveniencia más cercana se ubica a más de $1 \mathrm{Km}$.) identificadas como tiendas D.

Las tiendas participantes fueron elegidas con base en la recomendación del director de mercadotecnia de la cadena detallista según los perfiles identificados; cabe aclarar que todas las tiendas estudiadas están ubicadas en la zona metropolitana de la ciudad de Toluca, aunque la cadena cuenta con tiendas en varios municipios de la zona centro del Estado de México. En la siguiente fase de la metodología se diseñó un cuestionario altamente estructurado que fue aplicado por encuestadores capacitados y supervisados por una de las autoras de este trabajo. En el diseño del cuestionario se tomó en cuenta que los clientes serían entrevistados durante o inmediatamente después de realizar su pago en cajas, por lo cual se propuso un cuestionario simple y breve, organizado en tres secciones cuyo contenido se describe en el cuadro 1. Cada encuestador registró el día y horario de aplicación de sus encuestas para fines de verificar la calidad del muestreo.

En una siguiente etapa, el cuestionario se aplicó a clientes seleccionados sistemáticamente de la siguiente manera: uno de cada cliente que ingresó a la tienda fue contactado y se le hizo la invitación a participar; convenida su participación se esperó al cliente en la línea de cajas para aplicarle la encuesta. El cliente respondió a la encuesta, ya sea mientras esperaba para hacer su pago o bien justo después de realizarlo. El total de clientes entrevistados se calculó tomando como tamaño de la población a la cantidad esperada de clientes por día según datos históricos proporcionados por el gerente de cada tienda participante. Por tienda se programaron 100 o 200 encuestas, con un mayor número de encuestas realizadas en las tiendas que ofertan surtido completo, pues éstas atienden a una mayor cantidad de clientes por semana. 


\section{Cuadro 1 \\ Estructura del cuestionario de evaluación del impacto de oos}

\begin{tabular}{|c|c|}
\hline Sección & Reactivos \\
\hline $\begin{array}{l}\text { Perfil } \\
\text { de compra } \\
\text { (5 reactivos) }\end{array}$ & $\begin{array}{l}\text { 1. Frecuencia de compra en tienda ( } 5 \text { categorías: } 1=\text { siempre compra en tienda, a } \\
5=\text { ocasionalmente realiza compras en esta tienda) } \\
\text { 2. Número de compras en tiendas detallistas }(5 \text { categorías: } 1=\text { menos de } 1 \text { vez al } \\
\text { mes a } 5=\text { más de } 3 \text { veces por semana }) \\
\text { 3. Día preferido para realizar compra }(1=\text { entre semana, } 2=\text { en fin de semana) } \\
\text { 4. Tipo de compra }(1=\text { familiar, } 2=\text { para surtir negocio }) \\
\text { 5. Cantidad típica que gasta durante una visita (expresada en } \$)\end{array}$ \\
\hline $\begin{array}{l}\text { Registro } \\
\text { de faltantes } \\
\text { (5 reactivos) }\end{array}$ & $\begin{array}{l}\text { 1. ¿Encontró todos los productos que buscaba? }(1=\text { Sí, } 2=\text { No) } \\
\text { 2. ¿Qué productos no localizó? (clasificados en alguna } 4 \text { categorías básicas: } 1= \\
\text { alimentos, } 2=\text { bebidas, } 3=\text { higiene y belleza, } 4=\text { productos para limpieza del } \\
\text { hogar. Otros faltantes se asignaron a la categoría } 5=\text { otros }) \\
\text { 3. ¿Reporta faltantes a la tienda }(1=\text { Sí, } 2=\text { No) } \\
\text { 4. ¿Qué hace cuándo no localiza los productos deseados? }(1=\text { sustituye, } 2= \\
\text { pospone compra, } 3=\text { compra artículo en otra tienda, } 4=\text { compra todos los } \\
\text { productos en su lista en otra tienda) } \\
\text { 5. Pregunta abierta para registrar comentarios u observaciones en relación con } \\
\text { faltantes }\end{array}$ \\
\hline $\begin{array}{l}\text { Datos } \\
\text { personales } \\
\text { (5 reactivos) }\end{array}$ & $\begin{array}{l}\text { 1. Género }(1=\text { femenino, } 2=\text { masculino }) \\
\text { 2. Estado civil }(1=\text { casado o vive con pareja, } 2=\text { soltero }) \\
\text { 3. Edad }(1=\text { menos de } 25 \text { años, } 2=25-35 \text { años, } 3=36-45 \text { años, } 4=46-55 \text { años, } 5 \\
=56-65 \text { años, } 6=\text { más de } 65 \text { años }) \\
\text { 4. Lugar de residencia }(1=\text { dentro de un } 1 \mathrm{~km} \text {. del sitio de ubicación de la tienda, } \\
2=\text { más de } 1 \mathrm{~km} \text {. }) \\
\text { 5. Tamaño de la familia (no. de familiares con los que vive) }\end{array}$ \\
\hline
\end{tabular}

Las encuestas programadas en cada tienda se aplicaron en diferentes días de la semana y horarios de servicio dependiendo de la densidad de clientes que realiza compras en cada periodo. Entre el $25-35 \%$ de las encuestas se aplicaron entre semana (de lunes a viernes) y el resto (65-75\%) en fin de semana (sábado y domingo); los horarios de aplicación fueron tanto en la mañana (30\%) — desde la apertura de la tienda hasta las 2 p.m. - como por la tarde (70\%) — de las 2 p.m. hasta el cierre de la tienda. Estas cuotas de clientes corresponden a la proporción de clientes que compra en la tienda en diferentes días y horarios, lo que asegura que la muestra se asemeja a la población al lograrse la captación de clientes con diferentes perfiles y patrones de compra.

Del total de clientes contactados a su ingreso en la tienda, menos del 10\% se negó a responder a la encuesta; por lo tanto, el total de cuestionarios recopilados (556) corresponde cercanamente al tamaño de muestra programado (600), si bien la tasa 
de respuesta difiere en cada tienda y va del $85 \%$ (tienda C) al 99.5\% (tienda B). El cuadro 2 muestra el total de encuestas realizadas en cada tienda, así como una descripción del segmento mayoritario de consumidores. Este perfil está definido en términos de datos demográficos y de compra proporcionados por el mismo cliente durante la entrevista y validados por el gerente de la tienda con base en la información con la que cuenta sobre el perfil de su mercado. En particular el dato sobre el nivel socioeconómico del segmento principal de la tienda fue proporcionado por la gerencia; se eliminó del cuestionario la pregunta de ingreso debido a que se trata de una pregunta delicada que sólo fue respondida por dos de los clientes durante la prueba piloto.

\section{Cuadro 2}

\section{Total de encuestas aplicadas y perfiles de los encuestados en cada tienda}

\begin{tabular}{|c|c|c|c|}
\hline Tienda & $\begin{array}{l}\text { Total de } \\
\text { encues- } \\
\text { tas }\end{array}$ & $\begin{array}{l}\text { Perfil demográfico } \\
\text { del cliente }\end{array}$ & $\begin{array}{l}\text { Perfil de compra } \\
\text { del cliente }\end{array}$ \\
\hline $\begin{array}{l}\text { Tienda A = } \\
\text { surtido completo } \\
\text { con competencia } \\
\text { dentro de su área } \\
\text { de influencia }\end{array}$ & 175 & $\begin{array}{l}\text {-Mujeres casadas } \\
\text { jóvenes (25-35 años) } \\
-50 \% \text { residen dentro } \\
\text { del área de influencia } \\
\text {-Nivel socioeconómico } \\
\text { (SE) medio-bajo }\end{array}$ & $\begin{array}{l}\text {-61\% declara siempre comprar en esta } \\
\text { tienda } \\
\text {-No preferencia de compra en cierto día de } \\
\text { la semana } \\
\text {-Compra quincenal } \\
\text {-Monto mediano del ticket de compra es } \\
\text { de } \$ 325\end{array}$ \\
\hline $\begin{array}{l}\text { Tienda } \mathrm{B}= \\
\text { surtido completo } \\
\text { sin competencia } \\
\text { (aislada) }\end{array}$ & 199 & $\begin{array}{l}\text {-Mujeres casadas } \\
\text { jóvenes (25-35 años) } \\
\text {-60\% residen dentro } \\
\text { del área de influencia } \\
\text {-Nivel socioeconómico } \\
\text { (SE) medio }\end{array}$ & $\begin{array}{l}-71 \% \text { declara siempre comprar en esta } \\
\text { tienda } \\
\text {-Preferencia por compras en fin de semana } \\
\text {-Compra semanal } \\
\text {-Monto mediano del ticket de compra es } \\
\text { de } \$ 300\end{array}$ \\
\hline $\begin{array}{l}\text { Tienda } \mathrm{C}= \\
\text { conveniencia } \\
\text { con competencia } \\
\text { dentro de su área } \\
\text { de influencia }\end{array}$ & 85 & $\begin{array}{l}\text {-Mujeres casadas entre } \\
25-45 \text { años } \\
-76 \% \text { residen dentro } \\
\text { del área de influencia } \\
\text {-Nivel SE medio-alto }\end{array}$ & $\begin{array}{l}-59 \% \text { declara siempre comprar en esta } \\
\text { tienda } \\
\text {-Cierta }(56 \%) \text { preferencia de compra en } \\
\text { fin de semana } \\
\text {-Compra } 2-3 \text { veces por semana }(47 \%) \\
\text {-Monto mediano del ticket de compra es } \\
\text { de } \$ 200\end{array}$ \\
\hline $\begin{array}{l}\text { Tienda } \mathrm{D}= \\
\text { conveniencia } \\
\text { sin competencia } \\
\text { (aislada) }\end{array}$ & 97 & $\begin{array}{l}\text {-Mujeres casadas } \\
\text { jóvenes (25-35 años) } \\
-92 \% \text { residen dentro } \\
\text { del área de influencia } \\
\text {-Nivel SE medio-bajo }\end{array}$ & $\begin{array}{l}-71 \% \text { declara que siempre compra en la } \\
\text { tienda } \\
\text {-No preferencia de compra en cierto día de } \\
\text { la semana } \\
\text {-Monto mediano del ticket de compra es } \\
\text { de } \$ 80 \\
\text {-Compra al menos dos veces por semana } \\
(50 \%)\end{array}$ \\
\hline
\end{tabular}


Como se aprecia en el cuadro 2, la mayoría de los entrevistados (76\%) son personas que residen cerca de la tienda detallista, lo que concuerda con otros estudios sobre áreas de influencia para supermercados y tiendas de venta al menudeo (Kinner y Taylor, 1998) que indican que la mayor parte de la clientela de las tiendas detallistas eligen comprar en cierta tienda debido al beneficio de la cercanía. Se observan además diferencias en los patrones de compra por tienda, lo que valida la selección de tiendas y asegura la heterogeneidad en el perfil de los consumidores requerida para probar las hipótesis planteadas en este trabajo.

\section{Análisis de resultados}

Del total de 556 clientes entrevistados, 60 reportaron faltantes lo que corresponde a una tasa de oos del $10.79 \%$ a partir de la cual se estima con una confianza del 95\% que la verdadera tasa de faltantes observada por los clientes es algún número dentro del intervalo de 8.34 a $13.67 \%$. La tasa de faltantes que registra esta cadena detallista tiende a ser superior a la tasa de oos estimada por Gruen y Corsten (2002) y Gruen y Corsten (2008) a partir de la revisión de diversos estudios de oos, la cual se ubica entre el 5-10\%. El porcentaje de faltantes registrado por los clientes varía con la tienda, la menor tasa $5.15 \%$ se registró en la tienda D (conveniencia sin competencia en el área), en tanto que la mayor, estimada en $18.59 \%$, se registró en la tienda B (surtido completo sin competencia en el área). El mayor porcentaje de faltantes se dio en la categoría de alimentos (4.58\% de los entrevistados reportaron faltantes) y el menor en la categoría de bebidas $(0.67 \%$ de los clientes registró faltantes); no se observó ninguna relación aparente entre el tipo de tienda y el nivel de oos.

Para verificar las hipótesis de investigación H1-H3 se estimó un modelo de regresión logística multinomial (logit) utilizando el software estadístico SPSS v. 15, todos los datos disponibles se usaron como entrada para ajustar el modelo. El modelo multinomial condicional logit, introducido por McFadden (1974) es una representación matemática de como los individuos eligen opciones —en este caso alternativas para cubrir las faltantes de un producto- dependiendo de la utilidad (Ui) percibida en las características de un producto o servicio y/o de las preferencias del consumidor según su perfil demográfico y de compra. El modelo logit tiene la siguiente estructura:

$$
\begin{gathered}
\pi_{\mathrm{i}}=\operatorname{Pr}(\text { elegir la opción } \mathrm{i} \text { ante el caso de una faltante })=\exp \left(\mathrm{U}_{\mathrm{i}}\right) / \sum \exp \left(\mathrm{U}_{\mathrm{j}}\right) \\
\text { Donde } \exp \left(\mathrm{U}_{\mathrm{i}}\right)=\mathrm{e}^{(\beta 0+\beta 1 \mathrm{X} 1+\beta 2 \mathrm{X} 2+\ldots+\beta \mathrm{k} \mathrm{xk}+\mathrm{Gi})}
\end{gathered}
$$


Los coeficientes $\beta$ son parámetros a estimar, $\varepsilon_{\mathrm{i}}$ es un término aleatorio que para el caso del modelo logit se asumen son i.i.d. Gumbel y las X representan en el estudio las características demográficas (género, estado civil, residencia y rango de edad), el nivel de lealtad del cliente hacia la tienda detallista y el valor usual de su ticket de compra. Con respecto a la variable lealtad, ésta fue operada a través del reactivo referente a la frecuencia de compra en la tienda (ver cuadro 1); un menor valor en la escala utilizada indica que el cliente favorece cierta tienda con respecto a otras opciones, lo que da evidencia de una mayor lealtad hacia el establecimiento. Por el contrario, valores altos en la escala son evidencia de un cliente que busca variedad $\mathrm{y}$ tiene poca lealtad hacia un establecimiento detallista en particular. La frecuencia de compra en cierta tienda resulta ser sólo una variable sustituta de la lealtad, pues autores como Oliver (1999) reconocen que la lealtad es un constructor multidimensional que implica varias fases, donde la acción (comprar en la tienda) es la fase última. Esta expresión conductual de lealtad es resultado de una evaluación (lealtad) cognitiva de las ventajas de comprar en cierto supermercado o tienda de conveniencia (ventajas entre las que figura la disponibilidad de los productos deseados). Este estudio propuso una medida simple de la lealtad del cliente que se vincula directamente con la ocurrencia de oos.

La comprobación de las hipótesis H4 y H5 se realizó mediante la comparación entre los cuatro tipos de tiendas en cuanto a las distintas alternativas de acción reportadas por los clientes ante la presencia de oos. El cuadro 3 resume los resultados, las entradas del cuadro son porcentajes de clientes dentro de cada tienda; por tanto, el total por renglón suma el $100 \%$. Se aplicó la prueba de ji-cuadrada a los datos del cuadro cruzada, el estadístico de prueba resultó altamente significante (jicuadrada $=152, \mathrm{gl}=9$ y P-valor $=0.000)$ concluyéndose que las reacciones de los clientes dependen o se asocian con las características de la tienda (conveniencia $v s$. surtido completo, con competencia y sin ella en el área). En cada celda del cuadro 3 se reportan entre paréntesis los residuos estandarizados que permiten deducir hasta donde los valores observados difieren sustancialmente de los esperados. La magnitud de estos residuos (superiores a +3 o inferiores a -3 ) permite identificar cambios de tienda en exceso para la tienda 3 (conveniencia con competencia, residuo de 6.176) y muy escasos para la tienda 1 (surtido completo con competencia, residuo de -3.647). Mientras que la tienda 4 registra un porcentaje notable de clientes que optan por la sustitución por sobre cualquier otra alternativa (residuo de 6.063). 


\section{Cuadro 3}

Reacciones de los clientes para las distintas tiendas

\begin{tabular}{ccccc}
\hline Tienda & $\begin{array}{c}\text { Sustituye } \\
\text { productos }\end{array}$ & $\begin{array}{c}\text { Pospone compra } \\
\text { de faltante }\end{array}$ & $\begin{array}{c}\text { Compra faltante } \\
\text { en otra tienda }\end{array}$ & $\begin{array}{c}\text { Cancela y cambia } \\
\text { de tienda }\end{array}$ \\
\hline 1 & $38(-0.207)$ & $20(-0.667)$ & $38(3.754)$ & $4(-3.647)$ \\
2 & $31(-1.939)$ & $28(1.716)$ & $27(0.803)$ & $14(0.029)$ \\
3 & $20(-2.835)$ & $28(1.140)$ & $12(-2.282)$ & $40(6.176)$ \\
4 & $80(6.063)$ & $8(-2.792)$ & $1(-4.308)$ & $12.47(-0.982)$ \\
\hline
\end{tabular}

\section{Discusión de resultados}

El cuadro 4 resume los resultados del ajuste al modelo logit descrito en la sección anterior; el modelo completo se declara altamente significante $(\mathrm{P}$-valor $=0.000)$ con respecto al modelo que considera sólo el intercepto sin ninguna de las variables de explicación (X). Para realizar el ajuste del modelo fue necesario especificar una opción de referencia contra la cual se comparan las otras alternativas de acción que tiene el cliente. En este caso las opciones del consumidor ante una faltante son: 1) sustituir productos, 2) posponer la compra, 3) comprar la faltante en otra tienda y 4) cancelar el total de la compra para adquirir todos los productos, incluyendo el faltante, en otra tienda. La última alternativa es la que más afecta a la cadena detallista porque no sólo se pierde el valor de la compra del producto faltante, sino de toda la lista de compra del cliente; esto a la larga puede llevar a optar por preferir comprar en una tienda con surtido equivalente con el fin de reducir visitas a tiendas que no cuentan con todos los productos deseados. Por tanto, esta alternativa 4 fue elegida como la opción de referencia.

\section{Cuadro 4}

\section{Información del ajuste del modelo logit}

\begin{tabular}{c|c|c|c|c}
\hline \multirow{2}{*}{ Modelo } & Criterio de ajuste & Prueba del Cociente de Verosimilitud \\
\cline { 2 - 5 } & $\begin{array}{c}\mathbf{- 2 ~ L o g} \\
\text { Verosimilitud }\end{array}$ & $\begin{array}{c}\text { Ji- } \\
\text { cuadrada }\end{array}$ & $\begin{array}{c}\text { Grados } \\
\text { de libertad }\end{array}$ & P \\
\hline Solo intercepto $\left(\beta_{0}\right)$ & 1236.511 & & & \\
Final & 1082.990 & 153.521 & 30 & .000 \\
Pseudos R-cuadrada & \multicolumn{3}{|c|}{ Bondad de ajuste } \\
Cox y Snell & 0.255 & Método & Ji-cuadrada (gl) & P \\
Nagelkerke & 0.275 & Pearson & $1139(1092)$ & 0.155 \\
McFadden & 0.112 & Deviance & $990(1104)$ & 0.988 \\
\hline
\end{tabular}


En el caso del modelo logit no es posible calcular un coeficiente de determinación como el de un modelo de regresión lineal; sin embargo, hay pseudos R-cuadradas que se interpretan de manera similar. En este caso estos índices no resultaron muy altos, indican que si bien las características del cliente explican alrededor de un $25 \%$ de sus reacciones, hay factores adicionales no considerados explícitamente en este estudio que también definen la conducta del consumidor ante los oos. Finalmente, el cuadro 4 reporta dos pruebas alternativas para probar el ajuste del modelo; en este caso a ambas pruebas concluyen que el modelo de efectos principales, resultado de proponer una combinación lineal de las variables de explicación, se ajusta adecuadamente a los datos (P-valores mayores a 0.15 asociados a los estadísticos de prueba alternativos).

Evaluada la calidad global del modelo ajustado, se analizaron los coeficientes de las variables de explicación para probar las hipótesis de trabajo. En el cuadro 5 se reporta el estadístico ji-cuadrada que corresponde a la diferencia entre los logaritmos de las funciones de verosimilitud entre el modelo completo (con todas las X) y un modelo reducido en el que se ha omitido el efecto de una variable particular. La hipótesis estadística corresponde a probar que el efecto de esa variable sobre la alternativa de acción del cliente es nulo. Para aquellas variables cuyos coeficientes resultaron estadísticamente significantes (ver columna valor P) se reporta también el intervalo de confianza para el denominado cociente de ventaja (odds ratio) el cual es igual a $\mathrm{e}^{\beta i}$. Dado que hay tres alternativas o distintas reacciones declaradas por el cliente aparte de la alternativa de referencia, existe un cociente de ventaja asociado con cada reacción.

De acuerdo con la información del cuadro 5, los efectos declarados significantes son: lealtad a la tienda, valor del ticket de compra, género del cliente y sitio de residencia. Esto da apoyo parcial a las hipótesis H1-H3 al demostrarse que las reacciones del cliente dependen en parte de su perfil demográfico (género y residencia), así como de su nivel de lealtad y el monto de su compra en la tienda. Para precisar si a mayor lealtad y valor del ticket de compra se reduce la intención del cliente de cambiar de tienda e ir a otro establecimiento a adquirir los faltantes o incluso toda la lista de compra, es necesario analizar a detalle los valores de los denominados cocientes de ventaja (odds ratio) para estos efectos. 


\section{Cuadro 5}

\section{Pruebas para los coeficientes de las variables de explicación del modelo logit}

\begin{tabular}{|c|c|c|c|c|c|c|c|c|}
\hline \multirow{2}{*}{ Efecto } & Estimado del & \multicolumn{7}{|c|}{ Pruebas de hipótesis e intervalos de confianza } \\
\hline & $\begin{array}{c}-2 \mathrm{Log} \\
\text { Verosimilitud } \\
\text { Modelo } \\
\text { reducido }\end{array}$ & $\begin{array}{c}\mathbf{J i} \\
\text { Cuadrada }\end{array}$ & gl & Valor $\mathbf{P}$ & \multicolumn{4}{|c|}{$\begin{array}{l}\text { Intervalo de confianza al } 95 \% \\
\text { para el Cociente de oportunidad }\end{array}$} \\
\hline \multirow{5}{*}{ Lealtad } & \multirow{5}{*}{1138.239} & \multirow{4}{*}{55.248} & \multirow{4}{*}{3} & \multirow{4}{*}{0.000} & \multicolumn{2}{|c|}{ Límite inf } & \multirow{2}{*}{\multicolumn{2}{|c|}{$\begin{array}{l}\text { Límite sup } \\
\text { Reacción }\end{array}$}} \\
\hline & & & & & Reacción & & & \\
\hline & & & & & 1 & 0.610 & 1 & 0.926 \\
\hline & & & & & & 1.155 & 3 & 1.000 \\
\hline & & & & & Reacción & & Rea & \\
\hline \multirow[t]{4}{*}{ Ticket } & \multirow{4}{*}{1092.538} & \multirow{4}{*}{9.548} & \multirow{4}{*}{3} & \multirow{3}{*}{0.023} & 1 & 0.997 & 1 & 0.999 \\
\hline & & & & & 2 & 0.999 & 2 & 1.001 \\
\hline & & & & & 3 & 0.999 & 3 & 1.001 \\
\hline & & & & & Reacción & & Rea & \\
\hline \multirow[t]{2}{*}{ Género } & \multirow{2}{*}{1102.227} & \multirow{2}{*}{19.236} & \multirow{2}{*}{3} & \multirow{2}{*}{0.000} & 1 & 0.194 & 1 & 0.619 \\
\hline & & & & & 2 & 0.212 & 2 & 0.763 \\
\hline Edo. civil & \multirow{2}{*}{1089.854} & \multirow{2}{*}{6.864} & \multirow{2}{*}{3} & \multirow{2}{*}{0.076} & & 0.121 & $J$ & $0.4 / 1$ \\
\hline \multirow[t]{2}{*}{ Residencia } & & & & & Reacción & & Rea & \\
\hline & \multirow[t]{2}{*}{1117.980} & \multirow[t]{2}{*}{34.990} & \multirow[t]{2}{*}{3} & \multirow[t]{2}{*}{0.000} & 1 & $\begin{array}{l}0.272 \\
0.171\end{array}$ & $\begin{array}{l}1 \\
2\end{array}$ & $\begin{array}{l}1.135 \\
0757\end{array}$ \\
\hline \multirow{2}{*}{ Edad } & & & & & 3 & 0.078 & 3 & 0.338 \\
\hline & 1093.840 & 10.849 & 15 & 0.763 & & & & \\
\hline
\end{tabular}

Reacción 4 = cancelar la compra total de producto ante faltantes es la categoría de referencia

En el caso del modelo logit, el coeficiente de la variable independiente (X) no tiene una interpretación per se, sino a través del cociente de ventaja, el cual indica el cambio relativo u oportunidad de que un individuo prefiera la opción (i) con respecto a la opción de referencia (j) cuando el cliente pertenece al grupo $X=2$ con relación a cuando pertenece al grupo $X=1$. Si la variable $X$ es cuantitativa, como en el caso de valor de ticket de compra o nivel de lealtad, el cociente de ventaja, representa el cambio en las ventajas u oportunidades de elegir i sobre $\mathrm{j}$ cuando $\mathrm{X}$ aumenta en una unidad.

Para el caso de las variables demográficas, el cociente de ventaja para el efecto de X3 = género, en la reacción 1 = sustituir productos se estima en 0.346 (IC en el cuadro 5 incluye este estimado puntual); esto indica que la probabilidad de que 
un cliente decida sustituir productos con respecto a la $\operatorname{Pr}$ (cliente cancele compra) es de 0.346 cuando el cliente es de género masculino $(\mathrm{X} 3=2)$, en tanto que $\mathrm{Pr}$ (sustituir)/Pr (cancelar) es 1 para las mujeres. En otras palabras, las oportunidades (chances) de que un hombre con relación a una mujer opten por sustituir la faltante en vez de ir a comprar a otra parte son de 34.6 a 100; es decir, que por cada cien mujeres que prefieren sustituir, sólo alrededor de 35 hombres optan por la alternativa de sustitución sobre la de cancelación de su compra en la tienda debido a las faltantes registradas. El cociente de ventaja para las otras alternativas de acción ante faltantes (posponer compra o adquirir sólo el faltante en otra tienda) es también menor de uno, lo que demuestra que los hombres son más propensos que las mujeres a cambiar de tienda cuando registran faltantes de productos. Para la otra variable demográfica con coeficiente significante, $\mathrm{X} 5=$ residencia del cliente ( 1 = vive dentro de área de influencia de la tienda, $2=$ vive lejos de la tienda), el cociente de ventaja se declaró menor de uno para las reacciones posponer compra del faltante (reacción 2) y comprar producto faltante en otra tienda. De esta forma se concluye que los clientes que residen fuera del área de influencia de la tienda son más propensos a cambiar de tienda que a postergar la compra o visitar múltiples tiendas; esto es relativo a aquellos clientes que residen cerca de la tienda. Sin embargo, ambos tipos de clientes tienen preferencias similares por la sustitución de productos con relación a cancelar su compra completa y acudir a otra tienda a surtir el total de los productos deseados.

En cuanto al efecto de valor del ticket de compra para la alternativa de sustitución de productos se concluye que el verdadero cociente de oportunidad es menor de uno puesto que el intervalo de confianza (IC) va de 0.997 a 0.999 . A un incremento en el valor del ticket de compra corresponde un (mínimo) decremento en la oportunidad de que el cliente sustituya el producto faltante con respecto a cambiar a otra tienda para adquirir todos los productos de su lista de compra. Para las otras dos alternativas (sustitución, compra sólo de la faltante en otra parte) el IC incluye 1, en donde no hay preferencia para estas alternativas sobre la opción de cancelar compra y cambiar a otra tienda. Estos resultados no apoyan a la $\mathrm{H} 2$; una posible explicación para este resultado es que cuando un cliente adquiere una mayor cantidad de productos espera que el costo incurrido sea compensado con una mezcla de surtidos completa. Cuando no es el caso, su preferencia por la tienda cambia bruscamente y prefiere realizar su compra total en una alternativa mejor surtida.

En caso de lealtad, el verdadero cociente de ventaja, para la opción sustituir con respecto a cancelar la compra e ir a otra tienda, se declara inferior a uno (IC va 
de 0.610 a 0.926). Por tanto, si se incrementa la lealtad del cliente a la tienda (X toma menores valores según la escala utilizada), aumenta la oportunidad de que éste prefiera sustituir productos sobre la opción de cancelar su compra debido a la faltante registrada; por lo tanto, se tiene apoyo empírico para la H3.

El cuadro 3 es relevante para apoyar las hipótesis H4 y H5 como se indicó en la sección anterior, la prueba de ji-cuadrada y las entradas de este cuadro revelan diferencias significantes entre tiendas. Sin embargo, las tiendas difieren con relación a dos factores críticos: el tipo de surtido y la competencia dentro de su área de influencia. Para separar el efecto de estos factores se realizaron análisis de varianza (ANDEVA), por lo que se tomaron como respuestas a las siguientes variables: $\mathrm{Y} 1=$ $\%$ de clientes que declara comprar sólo los productos faltantes en otra tienda y Y2 $=\%$ de clientes que cancela el total de su compra cuando hay faltantes y opta por adquirir todos los productos de su lista de compra en otra tienda. En el cuadro 6 se presentan los resultados de este análisis.

\section{Cuadro 6 \\ Análisis de varianza para porcentaje de clientes que cambia de tienda}

\begin{tabular}{|c|c|c|c|c|c|}
\hline & & \multicolumn{2}{|c|}{$\begin{array}{l}\text { Variable } \mathrm{Y} 1 \text { = compra } \\
\text { faltante en otra tienda }\end{array}$} & \multicolumn{2}{|c|}{$\begin{array}{l}\text { Variable } \mathrm{Y} 2 \text { = cancela } \\
\text { compra y cambia tienda }\end{array}$} \\
\hline Fuente de variación & gl & $\mathrm{F}$ & $\mathrm{P}$ & $\mathrm{F}$ & $P$ \\
\hline Tipo de surtido & 1 & $11,475.77$ & 0.006 & 0.63 & 0.572 \\
\hline Competencia en área & 1 & $2,040.03$ & 0.014 & 0.22 & 0.719 \\
\hline \multirow[t]{2}{*}{ Error } & 1 & & & & \\
\hline & & R-cuadrada & $99.98 \%$ & R-cuadrada & $46.13 \%$ \\
\hline
\end{tabular}

Dado que sólo se cuenta con cuatro datos de porcentaje (uno para cada tienda), se asumió un modelo lineal aditivo para el cual se tiene un grado de libertad para estimar el error. Si bien esto disminuye la potencia de la prueba F en el ANDEVA, los valores de este proceso estadístico de prueba resultaron considerablemente grandes para el caso de Y1, lo que garantiza la identificación de diferencias significativas entre las tiendas, apoyando las hipótesis H4 y H5. Se cuenta además con los datos del cuadro 3 que concuerdan con los resultados del ANDEva. Para establecer cuáles tipos de tienda resultan más afectadas por la ocurrencia de oos se calculó para cada tipo de tienda el porcentaje de clientes que declararon compran el producto faltante en otro comercio. Para el factor competencia dentro del área de influencia se encontró que en las tiendas relativamente aisladas (B y D) sólo un 13.9\% de los clientes compra los faltantes en otro sitio, mientras $24.7 \%$ de clientes compran en 
tiendas con competencia cercana (A y C). Esto permite concluir que los clientes de aquellas tiendas que no tienen competencia dentro de su área de influencia tienden menos a comprar en otro establecimiento y favorecen más la sustitución (caso particular de la tienda 3). Con este resultado se da sustento empírico a la hipótesis H4. En relación con el factor tipo de surtido se encontró que $6.5 \%$ de los clientes de supermercados (tiendas A y B) optan por comprar el faltante en otro establecimiento en comparación con un $32.2 \%$ de los clientes de tiendas de conveniencia (C y D). En consecuencia se verifica también la hipótesis H5.

\section{Conclusiones}

Uno de los objetivos fundamentales de la administración de la demanda en el sector de venta al menudeo es asegurar la disponibilidad de productos en anaquel; sin embargo, ni la tecnología, ni la aplicación de estrategias de coordinación para el abasto han logrado eliminar la ocurrencia de faltantes en anaquel. Los resultados de una investigación realizada con clientes de una cadena de comercio al menudeo en formato de tienda de autoservicio, que opera en la región centro del Estado de México, indican que la tasa de faltantes en anaquel supera al 10\% reportado para estudios en otros países y asciende casi al $20 \%$ en algunas de las tiendas de la cadena. Asimismo, se registraron cuatro tipos de reacciones o alternativas de comportamiento de los clientes ante la ocurrencia de faltantes: a) sustituir productos por otros de misma marca o marcas diferentes; b) postergar la compra del faltante para otra visita a la tienda; c) adquirir los productos disponibles en la tienda y comprar faltantes en otra tienda; y d) cancelar la compra y adquirir el total de los productos requeridos en otra tienda. De estas reacciones, las últimas dos afectan directamente a la tienda detallista porque representan en el corto plazo una pérdida en ventas y en largo plazo un deterioro en la satisfacción y lealtad de los clientes.

El ajuste de un modelo logit multinomial permitió identificar el perfil demográfico de los clientes más sensibles al oos, los clientes de género masculino y aquellos que residen fuera del área de influencia de la tienda son quienes con más frecuencia cancelan su compra si no localizan los productos deseados. Si bien este segmento de clientes no es el principal para la cadena detallista (el segmento principal son las mujeres que residen cerca de la tienda), es relevante que aquellas tiendas que sirvan a consumidores con este perfil realicen esfuerzos adicionales por ofrecer la mezcla de productos que demandan. Contrario a lo esperado, el aumento en el valor del ticket de compra no incrementa la proporción de clientes que prefiere sustituir respecto a cambiar de tienda. 
El modelo logit permitió demostrar además que la tendencia a cambiar de tienda decrece a medida que aumenta la lealtad del cliente. Esto sugiere que la presencia de atributos negativos (ocurrencia de oos) si bien puede crear un estado temporal de insatisfacción, no es una condición suficiente para dejar de ser leal. Sin embargo, dado que en los supermercados se realizan compras de productos de consumo es difícil avanzar hacia la fase de lealtad afectiva (Oliver, 1999), por lo cual las tiendas detallistas tendrán que reforzar sus acciones de fortalecimiento de la lealtad cognitiva, lo que implica entre otras cosas garantizar la disponibilidad de productos; esto no excluye el promover la formación de una lealtad afectiva no sólo a través de satisfacer las demandas del cliente, sino también al hacer grata su experiencia de compra.

Análisis estadísticos adicionales (ji-cuadrada y ANDEVA) permitieron identificar las tiendas más afectadas por el desabasto; el porcentaje de clientes que ante un oos opta por adquirir en otra tienda los productos faltantes o incluso el total de su lista de compras se incrementa cuando la tienda es del tipo de conveniencia y también cuando hay otras tiendas con una oferta de surtidos similar localizadas en un área cercana.

Los resultados de esta investigación contribuyen a la literatura sobre oos en el contexto de tiendas regionales mexicanas y proporciona información sobre los segmentos de clientes y tipos de tiendas que son más afectados por el oos. La principal limitante de este estudio es que se trabajó con una única cadena detallista de cobertura regional y que opera sólo en los municipios del Estado de México. Extensiones relevantes a este trabajo incluyen estudios en otras cadenas mexicanas de cobertura nacional que operan en otras regiones del país; valoración del impacto de oos considerando distintas categorías de productos; determinación del grado de resistencia que tiene la lealtad de un cliente a la ocurrencia de faltantes, esto es evaluar por cuanto tiempo el cliente continúa comprando en una tienda que registra faltantes en anaquel antes de que tome la decisión de cambiar de manera definitiva a otro establecimiento. La realización de estos estudios es relevante para generalizar resultados y precisar cómo las actividades de abasto afectan el servicio, la satisfacción y la lealtad del consumidor mexicano. 


\section{Referencias}

Angerer, A. (2004). The impact of automatic store replenishment on retail: technologies and concepts for the out-of-stock problem. Wissencschaft: Gabler Edition.

Broniarczyk, S. M., W. D. Hoyer y L.McAlister (1998). Consumer's perceptions of the assortment offered in a grocery category: the impact of item reduction. Journal of Marketing Research, 35 (mayo), 166-176.

Corsten, D. y T.W. Gruen (2004). Stock-outs cause walkouts. Harvard Business Review, mayo, 26-28.

Chopra, S. y P. Meindl (2004). Supply Chain Management. 2a. ed. Upper Saddle River: Prentice Hall.

Dreze, X. S. J. Hoch y M. E. Purk (1994). Shelf Management and Space Elasticity. Journal of Retailing, 70 (4), 139-176.

Erosa Martín, V. E. y P. E. Arroyo López (2003). La anatomía de una faltante de anaquel en México. En Una revolución de negocios: adopción y uso de procesos electrónicos en México. Dirección de Investigación de la Asociación Mexicana de Estándares para el Comercio Electrónico (editor). México, 134.

Gruen, T. W. y D. Corsten (2002). Rising to the Challenge of out-of-stocks. ECR Journal, 2 (2), 45-58.

(2008). A Comprehensive Guide to Retail Out-of-Stock Reduction in the Fast-Moving Consumer Goods Industry, Washington, DC: Grocery Manufacturers of America, ISBN: 978-3-905613-04-9; http://www.nacds. org/user-assets/pdfs/publications/OutofStock.pdf; 31 de julio de 2009.

Kano, H., F. Takahashi y F. Tsuji (1984). Calidades atractivas y calidad obligatoria. Hiushitsu (Quality). The Journal of Japanese Society of Quality Control, 14 (sep.), 39-48. 
Kristensensen, K., H. Juhl y P. Ostergaard (2001). Customer satisfaction: some results for European retailing. Total Quality Management, 12 (7/8), 890-897.

Krum, F. (1994). Quantum leap. Progressive Grocer, January, 41-43.

Oliver, R. (1999). Whence consumer loyalty? Journal of Marketing, 63, 33-44.

Mc Fadden, D. (1974). Conditional logit analysis of qualitative choice behavior. En P. Zarembka (Ed.), Frontiers in Econometrics. Nueva York: Academic Press.

Retail Insight (2009). "Fixing the Basics - Onshelf Availability." http://www. retailinsight.uk.com/download/Fixing\%20the\%20Basics\%20-\%20OnShelf \%20Availability.pdf; 30 de julio de 2009.

Verbeke, W., P. Farris y R.Thurik (1998). Consumer response to the preferred brand out-of-stock situation. European Journal of Marketing, 32 (11/12), 1008-1028.

Van Woensel, T., Karel Van Donselaar, R. Broekmeulen y J. Fransoo (2007). Consumer responses to shelf out-of-stocks of perishable products, International Journal of Physical Distribution \& Logistics Management, 37 (9), 704-718 
